\title{
Notas para el diseño metodológico en el estudio de la movilidad social y las trayectorias laborales
}

\section{Notes for the Methodological design of a research in social mobility and labor trajectories}

\author{
Cristian Segura-Carrillo \\ cristian.segura@uab.cat \\ Departamento de Trabajo Social. Universidad \\ Autónoma de Barcelona, España / Universidad de \\ Concepción, Chile
}

Recepción: 10 Mayo 2020

Aprobación: 30 Noviembre 2020

Publicación: 01 Febrero 2021

Cita sugerida: Segura-Carrillo, C. (2021). Notas para el diseño metodológico en el estudio de la movilidad social y las trayectorias laborales. Cuestiones de Sociología, 24, e116. https://doi.org/10.24215/23468904e116
Resumen: El objetivo de esta nota metodológica es mostrar una perspectiva innovadora en el diseño de una investigación que tiene como eje analítico la movilidad social y las trayectorias laborales. Asimismo, se integra una visión de la segmentación del mercado laboral más contemporánea y se plantea el curso de vida como perspectiva teórico-empírica. Desde lo metodológico defendemos la potencia de un método mixto secuencial, con preponderancia en lo cuantitativo, y desde lo cualitativo creemos en la fuerza de la técnica biográfica-narrativa. Cada una de las fases será articulada por el parangón metodológico. Las reflexiones finales nos invitan a mirar el fenómeno de la movilidad social desde la potencia de los métodos mixtos, ya que permiten mayor comprensión y profundización de los fenómenos sociales. Sin embargo, nos hace reflexionar sobre la distancia que puede haber entre un potente diseño y las dificultades propias del trabajo empírico-analítico.

Palabras clave: Diseño Mixto, Trayectoria Laboral, AMOSIT, Movilidad social.

\begin{abstract}
The objective of this methodological note is to show an innovative perspective in the design of research that has social mobility and labor trajectories as its analytical axis. Likewise, it integrates a vision of the most contemporary labor market segmentation and considers the course of life as a theoreticalempirical perspective. From the methodological point of view, we defend the power of a mixed sequential method, with a predominant focus on the quantitative, and from the qualitative field we believe in the strength of the biographical-narrative technique. Each one of the phases will be articulated by the methodological paragon. The final discussions invite us to look at the phenomenon of social mobility from the power of mixed methods, since they allow greater understanding and deepening of social realities. However, it makes us wonder about the distance that can exist between a powerful design and the difficulties inherent to empirical-analytical work.
\end{abstract}

Keywords: Mixed Design, Labor Trajectories, AMOSIT, Social Mobility. 


\section{Introducción}

$\mathrm{El}^{1}$ diseño de investigación que se propone en esta nota metodológica tiene como finalidad profundizar en las dinámicas de transmisión intergeneracional de las desigualdades sociales en Chile y España. Corresponde a una investigación en curso de una tesis doctoral del departamento de Sociología de la Universidad Autónoma de Barcelona. Este estudio se inscribe, en primer lugar, bajo el abordaje del modelo de análisis AMOSIT (Modelo Analítico de Desigualdades Sociales y Trayectorias) para los años 2016-2019² y, en segundo lugar, al proyecto sobre Dinámicas de Movilidad Social en España (DINAMOS) financiado por el Ministerio de Ciencia, Innovación y Universidades de España para los años 2020-2022.

\section{1.- Desigualdad social, movilidad social y trayectorias laborales}

La desigualdad social se ha estudiado por mucho tiempo desde distintas perspectivas, destacando la preeminencia de una mirada desde el ámbito económico (generalmente a través del índice de GINI). Sin embargo, existen enfoques actuales que cuestionan esa forma de analizar las diferencias. Es por ello que desde las ciencias sociales se ha establecido, como preocupación central, realizar análisis de la desigualdad social desde enfoques más multidimensionales, ya que esto aporta a una mayor y mejor comprensión del esfuerzo que realizan los Estados para mejorar el nivel de desarrollo de un país.

En contraposición, el discurso de la igualdad en algunos escenarios se ha utilizado para lograr un cambio de paradigma en las sociedades postindustriales, dicho enfoque patrocina elementos de la equidad y la justicia social en la población en general (Colmenares, 2018). Dubet (2011) estudia la igualdad desde la justicia social, y refiere que hay dos formas de medir este enfoque en una sociedad: en base a las posiciones sociales y en razón a las oportunidades a las que pueden acceder los individuos. Para el primer razonamiento se busca "hacer que las distintas posiciones estén, en la estructura social, más próximas las unas de las otras, a costa de que entonces la movilidad social de los individuos no sea ya una prioridad" (Dubet, 2011, p. 9). El segundo enfoque expresa que la meritocracia y el esfuerzo son la clave para que las personas puedan moverse en las distintas posiciones sociales que existen en la sociedad (Dubet, 2011; Rawls, 1995). Sin embargo, no debe considerarse como máxima que las diferencias implican instintivamente desigualdad; solo serán más o menos perjudiciales en la medida en que se consoliden en la estructura social. Por ende,

Entendemos que las desigualdades sociales son la expresión de ciertas diferencias sociales observables en términos de posiciones jerárquicas, de acuerdo a los valores establecidos en una sociedad. Implican distribuciones desiguales de acceso a recursos (económicos, educativos y culturales, relacionales, de salud, etc.), oportunidades, prestigio o poder, que opera a través de mecanismos que dependen de ciertos rasgos sociales (clase, género, raza, etnia, edad, etc.), estableciendo la base de sistemas de estratificación social institucionalizados (López-Roldán \& Fachelli, 2020, p. 5).

En definitiva, la desigualdad está asociada a relaciones jerárquicas y de poder, dotando a algunos con ciertos privilegios en detrimento de otros. Las 
condiciones adscriptivas operan como elementos justificativos de la desigualdad, transformándose en un derecho para unos pocos, que se traducen en propiedades, mejor nivel de vida, acceso a activos, influencia y una condición distinta de ciudadanos (CEPAL, 2018). Esta cultura del privilegio erosiona los elementos de la meritocracia y el esfuerzo individual que pudiese realizar cualquier persona, ya que muchas de las acciones son ratificadas, validadas y defendidas por una institucionalidad y actores que se benefician de las reglas y prácticas establecidas en el juego desigual (CEPAL, 2018).

Guzmán, Barozet y Méndez (2017), autoras chilenas, han encontrado en sus investigaciones que una persona de un origen social de baja cualificación debe alcanzar sus metas en base a esfuerzo y mérito, convirtiéndose en un mecanismo de movilidad social. No obstante, para aquellos/as que tienen un origen social de mejor cualificación, las conexiones sociales, la formación de creencias y la generación de ciertas habilidades y capacidades, la formación de preferencias y aspiraciones, al parecer tendrían mayor peso al momento de elegir una carrera universitaria o ingresar al mundo laboral (Martínez, 2013). Son estas características adscriptivas las que pueden fomentar posiciones desiguales basadas en el origen social, dando paso a la desigualdad de oportunidades, ya que unos tendrán que esforzarse "más" para alcanzar mejores posiciones sociales, en contraste con aquellos que con el mínimo de esfuerzo les alcanzará para mantener la posición y el statu quo.

Nuestro análisis para el presente estudio se basa en la movilidad social, uno de los ejes clásicos de la sociología que, en cierta forma, aporta a la comprensión de la desigualdad. Permite conocer cómo se estructura una sociedad y la forma de redistribución de las desigualdades entre sus miembros y entre generaciones (padres-madres/hijos-as). Busca conocer cómo cambia la constitución social en razón de las oportunidades que ofrece a sus miembros. Para Hout (1988, en Dalle, 2016) el análisis de tendencias en la movilidad social en un país puede reflejar y ayudar a comprender el proceso de cambio social que vive ese país, ya que evidencia las oportunidades que se posibilitan o se restringen, condicionadas en mayor o menor fuerza por el origen social de los individuos.

Es por ello que nos planteamos el estudio de la Movilidad Intergeneracional desde el ámbito educativo y ocupacional, en donde se observan los cambios a través de una generación a otra (padre-madre/hijo-a) y la movilidad intrageneracional que observa los cambios que una persona experimenta entre el primer empleo de su inserción al mundo laboral y el empleo actual (Kerbo, 2009). La vinculación de ambos tipos de estudio permite revisar las dinámicas sociales desde dos focos: en un primer acercamiento se realiza una mirada de corto plazo que muestra los cambios en el mercado laboral, y en el largo plazo se espera observar las tendencias de los cambios macrosociales.

Las investigaciones sobre movilidad social fueron impulsadas por la teoría de la modernización, supuesto que permite mirar a las sociedades "modernas" con estructuras más productivas, niños/as más educados/as; lo que supone que las personas más vulnerables reciban mayores beneficios. Sin embargo, los estudios intergeneracionales han colocado en pugna los postulados de la teoría, ya que han establecido que existe asociación entre origen y destino. En España se ha encontrado que el destino social está condicionado directamente por el origen (Gil-Hernández, Marqués-Perales, \& Fachelli, 2017); desde el 
espacio latinoamericano existe algo similar, lo que se conoce como la cultura del privilegio, definida como aquel rasgo histórico que naturaliza las diferencias como desigualdades (esto en una lógica sociológica, correspondería a la teoría de la legitimización). Es decir que las "desigualdades se transmiten y reproducen en el presente” (CEPAL, 2018, p. 30).

El estado de los distintos análisis que se han realizado en el campo de la movilidad social se puede resumir en cinco periodos. Si bien se separan para otorgar mejor comprensión, esto no implica asumir que la discusión está cerrada en un periodo anterior y por eso se comenzó con el siguiente. Algunas formas de medición y concepción de la movilidad social siguen vigentes en la actualidad.

El inicio del debate comienza con Lipset y Zetterberg (1959), que hablaban de la similaridad entre países que han pasado el umbral de la industrialización. Sin embargo, con los análisis de Hout y Hauser en el año 1992, se encontró una variación estadísticamente significativa entre países respecto de las asociaciones origen-destino (Citado en Ricardi, 2017).

Un segundo periodo está dado por la tesis de la fluidez constante de Erikson y Goldtrorpe (1992), que comienza en el año 1992 que viene a contrarrestar las perspectivas más funcionalistas que expresan la existencia de una movilidad creciente. Estos autores plantean que los patrones de reproducción y movilidad en una sociedad se mantienen constantes en el tiempo, sin tener mucha variación entre las distintas cohortes (Dalle, 2016; Espinoza y Núñez, 2014; Ricardi, 2017).

Como tercera tesis se plantea que la movilidad depende de los tipos de estados de bienestar presentes en un país. Es así como Beller y Hout (2006) evidencian cómo las distintas políticas sociales o para la educación aportan a determinar los orígenes y destinos distintos y en perspectiva de mejora.

Posterior a ello, comienza la teoría liberal de la industrialización, con Blau y Duncan en la década del 70, sumados a autores como Parsons y Treiman que sostienen que los países industrializados tendrían tasas más altas de movilidad. Con posterioridad a esta teorización surgen algunos matices que tienen relación con que los procesos de industrialización potencian la movilidad ascendente, existe creciente igualdad de oportunidades, mayor homogeneización entre países y se generan sociedades más abiertas. Sin embargo, aun cuando la teoría ha sido muy productiva en sus lineamientos, las críticas que ha recibido también lo son: por un lado, se observa que el surgimiento de la teorización nace para un contexto particular, objetándole que tiene una visión un tanto reduccionista de la sociedad (Ricardi, 2017).

Luego surgen las teorías de la reproducción social y de la correspondencia, impulsadas para contrarrestar la mirada de los enfoques funcionalistas, explicitando la tesis máxima que dice que en las sociedades más industrializadas no se cumplen aspectos de la igualdad en las oportunidades y que la educación no sería clave en el proceso de apertura social. Se plantea que lo que sucede en las sociedades con enfoques capitalistas es un fuerte legado reproductivo de la estructura social, es decir que tiene un alto componente hereditario la posición de clase social que alcance el/la hijo/a (Bajoit, 2014; Sousa, 2010).

Uno de los elementos que permite reflejar la forma en que se mueven los/ as individuos en el espacio social es la medición de la fluidez, es decir las probabilidades que tienen las personas para moverse en el espacio social; algunos dirían que se basa en la igualdad de oportunidades presentes (Espinoza y Núñez, 
2014). Varios estudios en Europa y América latina han concluido que la hipótesis de la fluidez social común, la que dice que "los países comparten un patrón similar de movilidad", y el supuesto de la fluidez constante que "indica que la fluidez no varía a través del tiempo a pesar de los cambios en la industrialización”, van perdiendo fuerza en razón a las investigaciones empíricas realizadas (Featherman et al., 1975. Citado en Torche, 2015, p. 12). Este tipo de fluidez ha sido criticada en Europa y Estados Unidos, debido al surgimiento de nuevos métodos de análisis más sofisticados, en donde se ha encontrado que la educación superior se va universalizando y, por ende, la asociación origen/destino se vuelve más débil (Breen, 2004; Breen \& Karlson, 2014; Hout \& DiPrete, 2006; Marqués y Herrera-Usagre, 2010; Torche, 2015). Los análisis iniciales en Chile hablan de una sociedad que no es tan fluida, que tiende a la rigidez en sus patrones de movilidad, en contraposición a los países europeos que, según análisis recientes, son más móviles. Dicha característica sería indicativa de que las mujeres aportan mayor fuerza fluida a las mediciones (Bukodi \& Goldthorpe, 2016; Dalle, 2018; Espinoza y Núñez, 2014; Fachelli y López-Roldán, 2015 y 2017; MarquésPerales y Herrera-Usagre, 2010). Las conclusiones de los/as autores/as precisan que la educación sigue siendo un factor relevante en la inserción al campo laboral en los inicios de las trayectorias laborales, y eso marcaría el tipo de trayectorias que presentará una persona.

En la otra esfera de la movilidad social (la intrageneracional), los estudios han sido menos fructíferos; esto en general se debe a la falta de datos cuantitativos específicos que puedan medir longitudinalmente la entrada, transiciones y salidas del mercado de trabajo de una persona. Por ende, los estudios que se observan se realizan desde una variable proxy como la edad (Vallet, 2017; Pollack y Muller, 2018; Pfefer y Hertel, 2015, López-Roldán y Fachelli, 2018) y no desde el análisis específico de la movilidad social intrageneracional basada en el primer trabajo y el actual (Parrado, 2007). Existen acercamientos a dicha perspectiva, pero desde una mirada cualitativa, retrospectiva, con historias de vida que profundizan en los distintos procesos experienciales que vive una persona y las decisiones que toma para estar y vivir la historia que experimenta en el presente.

Dentro del análisis de la movilidad, uno de los aspectos que se ha tratado tradicionalmente es la variable género, que tiene una variante sexista, en la medida que solo ha considerado a los varones en la medición de la ocupación. Sin embargo, desde la década de 1980 se inicia una discusión respecto de esa mirada que permite que hoy se establezca el criterio de dominancia: es decir que se considera aquel padre/madre que tiene la mejor calificación ocupacional para tomarle como referencia en el análisis de la movilidad (sea tanto hombre como mujer). Dicho enfoque debe ser considerado en el análisis de este fenómeno, ya que resulta clave comparar a las mujeres de antaño con las contemporáneas y visibilizar así los cambios en la estructura social (Erikson, 1984; Fachelli y LópezRoldán, 2015).

En relación al término trayectoria laboral, debemos decir que este se analiza como el período de tiempo y los empleos que de forma sucesiva ha ido teniendo una persona desde su incorporación al mercado laboral hasta el momento actual. Autores como Miguélez y Torns (2011) han puesto énfasis en el estudio de las trayectorias en base al número de contratos, la rotación laboral y los cambios de la condición de la actividad (ocupación, desempleo, inactividad), cercano a los 
criterios de Mauro y Yañez (2005) frente a otras aproximaciones más orientadas hacia la "movilidad -ascendente o descendente- o la sucesión de posiciones ocupacionales y profesionales" (Arnal, Finkel, y Parra, 2013, p. 292).

La trayectoria laboral puede ser una guía de las oportunidades que genera una sociedad para sus miembros en edad activa. Uno de los elementos claves hoy en día -luego de que el modelo fordista fuera el de mayor peso en el mercado del trabajo- es que hay un aumento en trayectorias desestructuradas, con mayor temporalidad y crecimiento de la precariedad laboral. Es por ello que la educación en el campo laboral ha tomado fuerza; educación que los/as trabajadores/as deciden realizar con sus propios recursos, lo que ha llevado a que se evidencie un énfasis en el crecimiento de la formación en la empresa (patrocinada por la misma institución laboral) para enfrentar los cambios en las estructuras laborales, lo que ha repercutido en el desarrollo de trayectorias laborales con mayor linealidad y estructura, con más y mejores condiciones de empleo (Martín Artiles, Lope, Carrasquer y Molina, 2019).

En el mundo del trabajo, por mucho tiempo se consideró la teoría del Capital Humano como el enfoque que realzó la credencial educativa como único factor clave y suficiente para explicar el ingreso y sostenimiento de los/as sujetos/as en el mercado laboral. Sin embargo, hoy en día el solo hecho de la credencial educativa resulta insuficiente para la comprensión del mercado laboral. Es por ello que una variable contextual para observar las ocupaciones y las trayectorias es la segmentación del mercado de trabajo (SMT), ya que esta entrega una visión de mayor profundización al interpretar los movimientos y transiciones que tiene un individuo en las relaciones de trabajo en un contexto segmentado. Es por ello que la Teoría de la Segmentación Laboral aporta explicación en la estructuración de un mercado de trabajo que "no es homogéneo" y se diferencia en dos segmentos principales con una relación jerárquica entre ellos, entrelazados con diversos factores de oferta y de demanda que caracterizan cada uno de esos segmentos (López-Roldán, 1996; López-Roldán y Fachelli, 2019). Para esta investigación el enfoque heterodoxo de la teoría de la segmentación del mercado laboral resulta más cercano a los objetivos de la investigación: recopila tradición y experiencia de investigación realizada durante años por el QUIT ${ }^{3}$ (Lope, 2018; Martín-Artiles et al., 2018; Miguélez y Torns, 1998; Molina et al., 2019; Torns et al., 2011), cuestiona la mirada neoclásica de explicación del mercado laboral, en tanto pone el acento en el funcionamiento segmentado del mercado capitalista, aludiendo así a cierta cultura de acaparamiento del poder y dinámicas que potencian la desigualdad (López-Roldán y Fachelli, 2019; Recio, 2018). La teoría de la segmentación reconoce esas particularidades de los mercados del trabajo, trata de comprender aquellos factores que dan estructura a la división de los mercados y de explicar las nuevas formas de relaciones laborales, enmarcadas en condiciones, muchas veces, asociadas a la precarización del trabajo. El nuevo enfoque de la segmentación del mercado laboral propuesto por Grimshaw, Fagan, Hebson \& Tavora (2017) reúne elementos claves de tres tradiciones teóricas que han demostrado ser valiosas en la articulación de las causas, características y consecuencias de las desigualdades en el trabajo y empleo. Los autores plantean tres elementos claves: en primer término, plantean una ruptura con la tradición teórica radical de la ortodoxia económica respecto del capital humano o diferencias en productividad. Su significado de larga data radica 
en su oposición a la economía neoclásica, que asume que los empleadores se ajustan automáticamente a los cambios en el lado de la oferta en educación y habilidad para que utilicen el potencial productivo en el mercado laboral. Sin embargo, las desigualdades no se fomentan solo en el lado de la oferta a través de reglas y convenciones sociales o culturales exógenas, también, y quizás de manera predominante, a través de políticas y prácticas institucionalizadas formales e informales en mercados laborales y lugares de trabajo (Grimshaw et al., 2017). Una segunda idea en este modelo de segmentación son las distinciones por género. Hay evidencia de que los empleadores explotan el perfil de género y las prácticas salariales en la creencia de que las mujeres están menos comprometidas con el trabajo que sus homólogos masculinos. Es probable que el valor productivo de los trabajos realizados predominantemente por mujeres esté infravalorado, ya que históricamente las mujeres han tenido menos posibilidades que los hombres para establecer un alto estatus para esas ocupaciones y sectores dominados por mujeres. De modo que para el mismo nivel de habilidad en los trabajos ocupados por mujeres, es más probable que se le atribuya el estatus de periferia y se pague a un nivel inferior a los realizados por hombres (Basu, 1990). La tercera área de la literatura que sustenta este foco de la segmentación del mercado laboral es la teoría institucionalista comparada. Esta tradición teórica se basa en la idea de que los mercados laborales están socialmente construidos, moldeados e influenciados por las instituciones y por los actores sociales (Grimshaw et al., 2017).

Es en este contexto laboral segmentado, donde la relación entre trayectoria laboral y movilidad social tienen sentido, ya que al analizar la trayectoria que experimenta una persona en su curso de vida se van leyendo movimientos en lo micro y macrosocial que pueden explicar el cambio o no en la movilidad intergeneracional; todo ello en un contexto más holístico del mercado laboral, que considera las interacciones dinámicas de la demanda y la oferta, integrando las relaciones capital-trabajo, así como el papel de las instituciones sociales y del género en la configuración del empleo (European Commission, 2019; Grimshaw et al., 2017). 


\section{AMOSIT y Orientación Metodológica}

\section{Gráfico 1}

Analytical Model on Social Inequalities and Trajectories (AMOSIT)

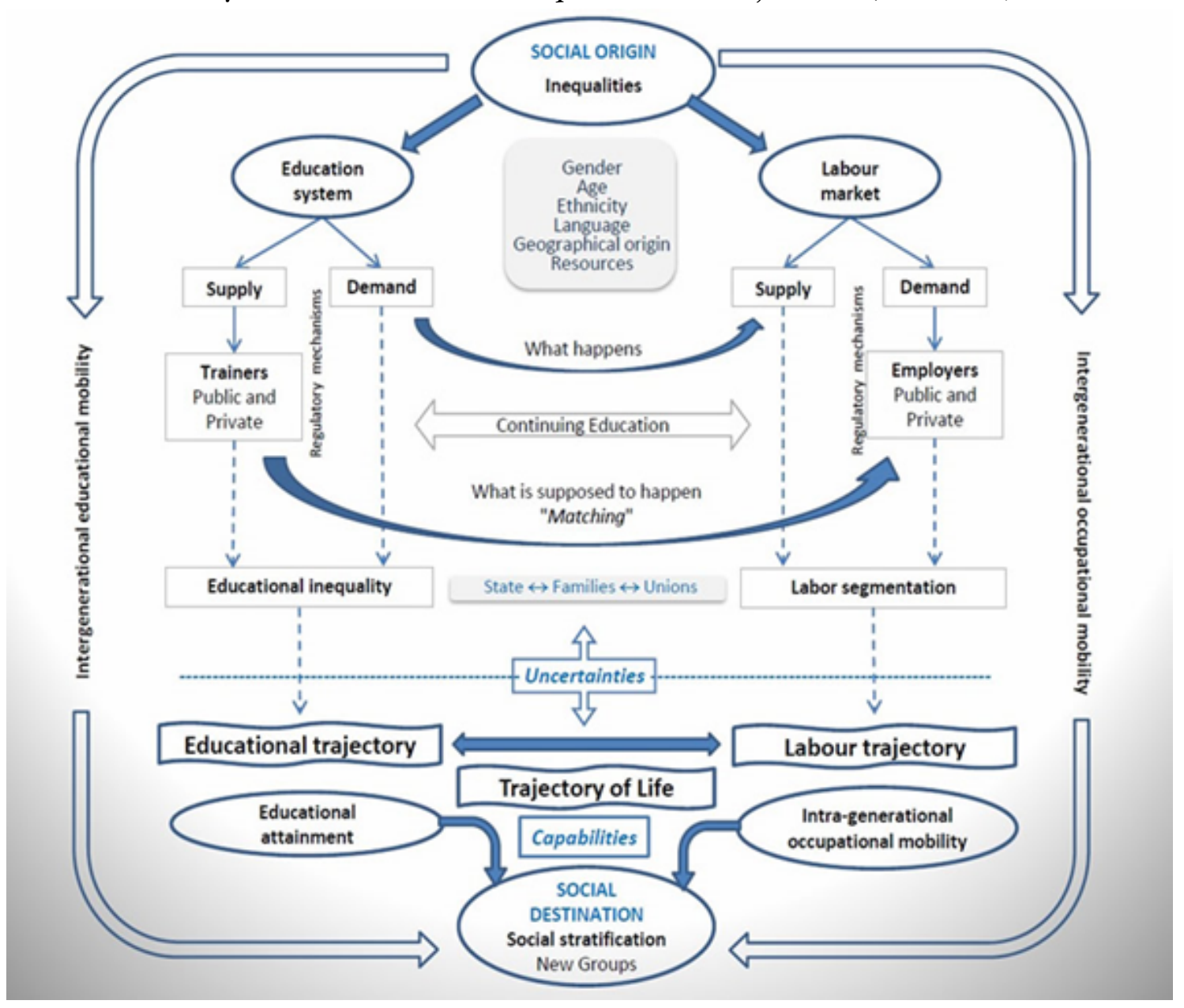

Fuente: López-Roldán y Fachelli (2017).

La perspectiva teórica de AMOSIT (Modelo Analítico de Desigualdades Sociales y Trayectorias) adquiere relevancia a la hora de generar discusión en la comprensión para la reducción de las desigualdades sociales, entendiendo que factores como el trabajo y la educación son claves para la movilidad social y deberían aportar a la reducción de desigualdades estructurales y sociales, al implicar que, a medida que una sociedad tiene más fluidez social, esta tiende a disminuir las desigualdades sociales (Fachelli y López-Roldán, 2017; Torche, 2015; Torche y Wormald, 2004).

El gráfico 1 presenta de forma sintética la articulación entre las distintas dinámicas del mercado educativo, laboral, el trabajo reproductivo y la vida cotidiana, que explican las desigualdades sociales desde las distintas posiciones sociales de partida que tienen los sujetos y los diferentes procesos e interrelaciones sociales que viven para llegar a su destino social actual. Es decir que la estratificación social es un ente regulador de las desigualdades en la medida en que se generan posiciones desiguales con acceso a recursos e interacciones distintas, lo que tiene como resultado un logro menor o mayor en educación que al mismo 
tiempo lleva a obtener peor o mejor posición laboral (López-Roldán y Fachelli, 2017 y 2020).

Desde el punto de vista metodológico, se integra parte del modelo AMOSIT; este tipo de estudio permite mayor profundización y comprensión de los procesos que estructuran las desigualdades ocupacionales. Creemos que debe ser desde una aplicación de método mixto, con un diseño secuencial anidado (Alcaide, Fachelli, y López-Roldán, 2019; Creswell y Plano, 2007; HamuiSutton, 2013; Hernández, Fernández, y Baptista, 2014; López-Roldán y Fachelli, 2015). Este diseño concibe dos etapas: una cuantitativa y otra cualitativa. En este encadenamiento se da centralidad a la fase cuantitativa (CUAN-cual), ya que tendrá un peso predominante en la investigación, mientras que el enfoque cualitativo será articulado con los resultados obtenidos en la fase cuantitativa, con el objetivo de profundizar en algunos aspectos, dinámicas y perfiles, y lograr así una comprensión más acabada del fenómeno.

El diseño mixto permite aprovechar las fortalezas de cada método para reducir sus limitaciones. Desde lo cualitativo se rescata la profundidad y comprensión del fenómeno y desde lo cuantitativo la generalización y comprensión específica que entregan los datos (Green et al., 2015). Por ende, esta metodología da cuenta de la interacción que existe entre lo micro y lo macro, y por consiguiente permite aproximarse a las condiciones normativas y estructurales de la sociedad, a la vez que posibilita revisar los discursos de los actores para observar las distintas representaciones que hacen de la realidad y de sus procesos de movilidad.

La fase cuantitativa se desarrolla en varios pasos. En primera instancia se analizará la movilidad intergeneracional, posterior a ello la movilidad intrageneracional en conjunto con las trayectorias laborales y la segmentación del mercado del trabajo. Para vincular perfiles muestrales entre la fase cuantitativa y cualitativa se utilizará la técnica del parangón metodológico, cuya fuerza radica en que nos permite definir perfiles tipo que surgen desde el análisis cuantitativo y que orientan la búsqueda y selección de la muestra para las entrevistas en la fase cualitativa (Alcaide et al., 2019).

En la fase cualitativa se realizarán entrevistas en profundidad, biográficas y narrativas, para observar los cursos de vida (Elder, Johnson, y Crosnoe, 2003; Elder y Giele, 2009; Muñiz Terra, 2012). Con esta técnica (biográfica) se busca que el/a hablante puede reconstruir su historia laboral y familiar, resaltando aspectos relevantes que la propia persona considera en su historia; para ello resultan clave las perspectivas de Pujadas (1990), Roberti (2012) y Bassi (2014), que orientan las formas en que se puede concebir "la cocina" del método, es decir, el hacer en el campo con los/as actores claves.

La fortaleza que tiene el método biográfico es que permite plantear un relato en profundidad de la historia vital de las personas. En nuestro caso esto se realiza con foco en las ocupaciones y con una mirada desde la adolescencia hasta el momento actual, para descubrir las transiciones, cambios laborales, y las opciones analizadas por la propia persona para llegar a las decisiones que tomó. Esto, no para analizar ni "leer" al hablante, más bien el relato biográfico posibilita que a través del/a narrador/a se nos muestre un mundo social desconocido (Bassi, 2014; Capriati, 2017; Güelman, 2013; Morales, 2016; Pujadas, 1990; Roberti, 2012; Rubilar, 2013; Serrano, Revilla y Arnal, 2016). 
Posteriormente a ambas fases se procede a un proceso de triangulación, en donde se enlazarán los resultados de las técnicas cuantitativas (perfiles de trayectorias laborales y movilidad social) con las historias de vida de los casos del ámbito cualitativo. El análisis será sistemático y detallado en cada uno de los perfiles y procesos sociales que evidencien las experiencias de las personas entrevistadas.

\section{Reflexiones finales}

El foco de esta investigación es analizar las dinámicas del cambio social y de transmisión de las desigualdades sociales observadas a lo largo del tiempo en términos de movilidad intergeneracional e intrageneracional, y las trayectorias laborales contextualizadas por la teoría de la segmentación del mercado del trabajo. Destacamos como planteamiento original de esta propuesta el correlacionar aspectos y variables que habitualmente son tratados de forma separada en una sola mirada de investigación. Asi, orientamos esta investigación hacia el análisis del vínculo entre cada variable utilizando un modelo innovador y contemporáneo (AMOSIT).

Valoramos inicialmente que los estudios que utilizan un método mixto en su diseño resultan innovadores, al tomar las potencialidades de lo cuantitativo y de lo cualitativo. Se resalta la posibilidad de utilizar insumos como el parangón metodológico para la selección de los sujetos para realizar entrevistas, técnica de reciente utilización, que permite entregar certeza respecto a los perfiles cualitativos y rescatar la tipología de sujetos más relevantes en cada territorio, y así profundizar en cada historia y tener un análisis discursivo de los comportamientos sociales a nivel macro.

Otro elemento a resaltar tiene que ver con su lógica comparada, ya que podría aportar insumos para la comprensión de los distintos tipos de movilidad al observar similitudes y diferencias en los contextos sociales chileno y español.

A partir del resultado de esta práctica de investigación cabrá plantear asimismo cómo se pueden aportar elementos de diagnosis y orientaciones para las políticas públicas con un objetivo de justicia social de disminuir las desigualdades sociales desde las potencialidades y rezagos que existen en cada territorio, tanto el español como el chileno.

Ahora queda preguntarse si este diseño -que tiene fuerza teórica y cuyas variables tratadas por separado presentan fuerza empírica- puede mostrar una realidad social específica y a la vez general de las estructuras sociales, institucionales y culturales de la desigualdad social en cada país con mayor profundidad y especificidad que los estudios que ya han analizado cada una de las variables por separado.

\section{Referencias}

Alcaide, V., Fachelli, S., \& López-Roldán, P. (2019). The Typological Paragon\#: A Methodological Proposal of Mixed Designs. Bulletin de Méthodologie Sociologique, 141, 64-84. https://doi.org/10.1177/0759106318813293

Arnal, M., Finkel, L., y Parra, P. (2013). Crisis, desempleo y pobreza: análisis de trayectorias de vida y estrategias en el mercado laboral. Cuaderno de Relaciones 
Laborales, 31(2), 281-311.https://doi.org/http://dx.doi.org/10.5209/rev_CRL A.2013.v31.n2.43221

Bajoit, G. (2014). Relaciones de clases y modos de reproducción: Teoría y Análisis. Cultura y Relaciones Sociales, 9(17), 9-52. Retrieved from http://www.scielo.org $. \mathrm{mx} / \mathrm{pdf} / \mathrm{crs} / \mathrm{v} 9 \mathrm{n} 17 / \mathrm{v} 9 \mathrm{n} 17 \mathrm{al} . \mathrm{pdf}$

Bassi, J. (2014). Hacer una historia de vida: Decisiones clave durante el proceso de investigación. Athenea Digital, 14(3), 129-170.

Basu, K. D. A. S. (1990). Flexible labour utilisation in the private service sector. Work, Employment \& Society, 4(4), 517-530. https://doi.org/10.1177/073998638700 92005

Beller, E., \& Hout, M. (2006). Welfare states and social mobility: How educational and social policy may affect cross-national differences in the association between occupational origins and destinations. Research in Social Stratification and Mobility, 24. https://doi.org/10.1016/j.rssm.2006.10.001

Breen, R. (2004). Social Mobility in Europe (R. Breen, Ed.). Print ISBN-13: 9780199258451/ DOI: https://10.1093/0199258457.001.0001

Breen, R., \& Karlson, K. B. (2014). Education and Social Mobility: New Analytical Approaches. European Sociological Review, 30(1), 107-118. https://doi.org/10.1 093/esr/jct025

Bukodi, E., \& Goldthorpe, J. H. (2016). Educational attainment - relative or absolute - as a mediator of intergenerational class mobility in Britain. Research in Social Stratification and Mobility, 43, 5-15. https://doi.org/10.1016/j.rssm.2015.01.00 3

Capriati, A. (2017). Tensiones y desafíos en el uso del método biográfico. Cinta de Moebio, 60, 316-327. https://doi.org/10.4067/S0717-554X2017000300316

CEPAL. (2018). La ineficiencia de la desigualdad. TRIGÉSIMO SÉPTIMO PERÍODO DE SESIONES DE LA CEPAL, 270. Retrieved from https://repositorio.cepal.o $\mathrm{rg} /$ bitstream/handle/11362/43442/6/S1800059_es.pdf

Colmenares, N. (2018). La dimensión territorial de la desigualdad social en Chile, desde la perspectiva de las politicas públicas para la superación de la pobreza. Universidad Complutense de Madrid.

Creswell, J., \& Plano, V. (2007). Choosing a mixed methods design. In J. Creswell \& V. Plano (Eds.), Designing and conducting; Mixed Methods Research (pp. 59-88). SAGE Publications Ltd.

Dalle, P. (2016). Movilidad social desde las clases populares: Un estudio sociológico en el Area Metropolitana de Buenos Aires (1960-2013). Retrieved from http://www.ii gg.sociales.uba.ar

Dalle, P. (2018). Climbing up a steeper staircase: Intergenerational class mobility across birth cohorts in Argentina (2003-2010). Research in Social Stratification and Mobility, 54, 21-35. https://doi.org/10.1016/j.rssm.2017.12.002

Dubet, F. (2011). Repensar la Justicia Social: contra el mito de la igualdad de oportunidades. In S. Veitiuno (Ed.), Repensar la justicia social. Retrieved from ht tp://www.sigloxxieditores.com.ar/pdfs/dubet_repensar_la_justicia_social.pdf

Elder, G. H., Johnson, M. K., \& Crosnoe, R. (2003). The Emergence and Development of Life Course Theory. Handbook of the Life Course, 3-19. https://doi.org/10.10 07/978-0-306-48247-2_1

Elder, J., G. H. G. H., \& Giele, J. Z. (ed.) (2009). Life course research: Development of a field. In The craft of life course research (pp. xii-372). https://dx.doi.org/10.413 5/9781483348919.n1 
Erikson, R. (1984). Social class of men, women and families. Sociology, 18(4), 500-514. https://doi.org/10.1177/0038038584018004003

Erikson R. \& Goldthorpe J. (1992) The Constant Flux. A Study of Class Mobility in Industrial Societies. Oxford: Clarendon Press.

Espinoza, V., y Núñez, J. (2014). Movilidad ocupacional en Chile 2001-2009. ¿Desigualdad de ingresos con igualdad de oportunidades? Revista Internacional de Sociologia, 72(1), 57-82. https://doi.org/10.3989/ris.2011.11.08

European Commission. (2019). New Visions for Gender Equality 2019 (N. Crowley \& S. Sansonetti, Eds.). https://doi.org/10.2838/00811

Fachelli, S., y López-Roldán, P. (2015). ¿Somos más móviles incluyendo a la mitad invisible? Análisis de la movilidad social intergeneracional en España en 2011. Revista Española de Investigaciones Sociologicas, 150, 41-69. https://doi.org/10.5 $477 /$ cis/reis. 150.41

Fachelli, S., y López-Roldán, P. (2017). La fluidez social de las mujeres modera la rigidez de los varones y de la inmigración. Revista Del Col.legi de Politólegs i Sociólegs de Catalunya. Retrieved from https://ddd.uab.cat/pub/artpub/2017/169786/amb polsoc_a2017m1d23a2iSPA.pdf

Gil-Hernández, C. J., Marqués-Perales, I., \& Fachelli, S. (2017). Intergenerational social mobility in Spain between 1956 and 2011: The role of educational expansion and economic modernisation in a late industrialised country. Research in Social Stratification and Mobility, 51, 14-27. https://doi.org/10.1016/j.rssm.2017.06.0 02

Green, C. A., Duan, N., Gibbons, R. D., Hoagwood, K. E., Palinkas, L. A., \& Wisdom, J. P. (2015). Approaches to Mixed Methods Dissemination and Implementation Research: Methods, Strengths, Caveats, and Opportunities. Administration and Policy in Mental Health and Mental Health Services Research, 42(5), 508-523. ht tps://doi.org/10.1007/s10488-014-0552-6

Grimshaw, D., Fagan, C., Hebson, G., \& Tavora, I. (Eds.). (2017). Making work more equal. Anew labour market segmentation approach. Manchester: Manchester University Press.

Güelman, M. (2013). Las potencialidades del enfoque biográfico en el análisis de los procesos de individuación. Revista Latinoamericana de Metodología de La Investigación Social, 3(5), 56-68. Retrieved from http://relmis.com.ar/ojs/index. $\mathrm{php} / \mathrm{relmis} /$ article/view/62

Guzmán, V., Barozet, E., y Méndez, M. (2017). Legitimación y crítica a la desigualdad: una aproximación pragmática. Convergencia, Revista de Ciencias Sociales, 24(73), 87-112. Retrieved from http://www.scielo.org.mx/pdf/conver/v24n73/1405-14 35-conver-24-73-00087.pdf

Hamui-Sutton, A. (2013). Un acercamiento a los métodos mixtos de investigación en educación médica. Investigación En Educación Médica, 2(8), 211-216. https://do i.org/10.1016/S2007-5057(13)72714-5

Hernández, R., Fernández, C., y Baptista, P. (2014). Metodología de la Investigación Sexta edición, Editorial McGRAW-HILL. México DF. ISBN: 978-1-4562-2396-0

Hout, M., \& DiPrete, T. A. (2006). What we have learned: RC28's contributions to knowledge about social stratification. Research in Social Stratification and Mobility, 24(1), 1-20. https://doi.org/10.1016/j.rssm.2005.10.001

Kerbo, H. (2009). Estratificación Social: El conflicto de clase en perspectiva histórica, comparada y global. Madrid: Mc GawHill. 
Lipset, S. M. \& Zetterberg H. (1959) Social mobility in industrial societies. Berkeley: University of California Press.

Lope, A. (2018). Limitaciones de la formación a las personas ocupadas para adecuar sus capacidades a los cambios en el empleo. In Fausto Miguélez (Ed.), La revolución digital en España. Impacto y Retos sobre el Mercado de Trabajo y el Bienestar (pp. 44). Barcelona. Retrieved from https://ddd.uab.cat/record/190326

López-Roldán, P. (1996). La construcción de una tipologia de segmentación del mercado de trabajo. Papers, Revista de Sociología, 48, 41-58.

López-Roldán, P., y Fachelli, S. (2015). Clasificación de las técnicas de análisis de datos. In Metodología de la Investigación Social Cuantitativa. Retrieved from https://dd d.uab.cat/pub/caplli/2015/131468/metinvsoccuan_cap3-5a2015.pdf

López-Roldán, P., \& Fachelli, S. (2017). Social Inequalities in Europe and Latin America. An Analysis Model Based on Life, Occupational and Educational Trajectories Research. In Working Paper Series. Retrieved from https://ddd.uab .cat/record/181855

López-Roldán, P., y Fachelli, S. (2019). Segmentación del empleo y apreciación de la educación en un modelo productivo anclado. Análisis comparativo entre España y Argentina. Papers. Revista de Sociologia, 104(2), 1. https://doi.org/10.5565/re v/papers. 2571

López Roldán, P., y Fachelli, S. (2019). Segmentación del empleo y apreciación de la educación en un modelo productivo anclado. Análisis comparativo entre España y Argentina. Papers. Revista de Sociologia, 104(2), 159. https://doi.org/10.5565/ rev/papers.2571

López-Roldán, P., \& Fachelli, S. (2021). A General Model for the Comparative Analysis of Social Inequalities Between Europe and Latin America. In P. López-Roldán \& S. Fachelli (Eds.), Towards a Comparative Analysis of Social Inequalities between Europe and Latin America (pp. 3-34). Cham: Springer International Publishing. https://doi.org/10.1007/978-3-030-48442-2_1

Marqués, I., y Herrera-Usagre, M. (2010). ¿Somos más móviles? Nuevas evidencias sobre la movilidad intergeneracional. Revista Española de Investigaciones Sociológicas, 131(2), 43-73. Retrieved from http://eds.a.ebscohost.com/eds/pdfviewer/pdfvie wer?vid=0\&sid=43741e5b-9e31-4f9d-8f97-e66a130f09a7\%40sessionmgr 4006

Martín-Artiles, A., Lope, A., Barrientos, D., Moles, B., Carrasquer, P., y Molina, O. (2018). Transición, Trayectorias y discursos. Análisis de Grupos de Discusión. In WORKING PAPER SERIES (No. 23; Centre d'E).

Martín Artiles, A., Lope, A., Carrasquer, P., y Molina, O. (2019). Aprendizaje basado en el trabajo: trayectorias laborales discontinuas. Cuadernos de Relaciones Laborales, 37(1), 203-229. https://doi.org/10.5209/crla.63826

Martínez, J.S. (2013). Estructura Social y Desigualdad en España. Ediciones Catarata.

Miguélez, F. y Torns, T. (1998). Introducción al análisis del trabajo y de la vida cotidiana. Papers. Revista de Sociologia, 55, 9. https://doi.org/10.5565/rev/pape rs. 1930

Molina, O., Verd, J. M., Cruz, I., Palauskaite, E., Dumčius, R., Venckute, M., ... VacasSoriano, C. (2019). Labour market change, Labour market segmentation\#: Piloting new empirical and policy analyses. https://doi.org/10.2806/30796

Morales, C. (2016). La investigación biográfico-narrativa: easpectos metodológicos o metódicos? Investigium IRE: Ciencias Sociales y Humanas, VII(1), 132-141. http ://dx.doi.org/10.15658/CESMAG16.050701010 
Muñiz Terra, L. (2012). Carreras y trayectorias laborales: una revisión crítica de las principales aproximaciones teórico - metodológicas para su abordaje. Revista Latinoamericana de Metodología de las Ciencias Sociales, 2(1), 35-65.

Parrado, E. (2007). La reestructuracio\#n econo\#mica y la movilidad intrageneracional de clase en Me\#xico. En F. Cortés, A. Escobar y P. Solís, (coord.) (eds.), Cambio estructural y movilidad social en Me\#xico (pp. 163-221). Mexico: El Colegio de México.

Pujadas, J. J. (1990). El método biográfico en sociología. En Cuadernos metodológicos. Centro de Investigaciones Sociológicas.

Rawls, J. (1995). Teoria de la Justicia. The belnap Press of Harvard University.

Recio, A. (2018). Desigualdades en el mercado laboral. En F. Miguelez (ed.), La revolución digital en España. Impacto y Retos sobre el Mercado de Trabajo y el Bienestar (pp. 185-211). Centre dÈstudis Sociològics sobre la Vida Quotidiana i el Treball, Universitat Autònoma de Barcelona.

Ricardi, C. (2017). La movilidad social y educativa de las generaciones jóvenes\#: una perspectiva comparada entre Europa y América Latina. 369. Retrieved from https://cataleg.uab.cat/iii/encore/plus/C__Smovilidadsocialandchileande spaña_Orightresult_U_X 0 ?lang=cat\&link $=$ https $\% 3 \mathrm{~A} \% 2 \mathrm{~F} \% 2$ Flogin.are.ua b.cat\%2Flogin\%3Furl\%3Dhttp\%3A\%2F\%2Fsearch.ebscohost.com\%2Flogin.as px\%3Fdirect\%3Dtrue\%26site\%3Deds-live\%26db\%3Ded

Roberti, E. (2012). El enfoque biográfico en el análisis social: claves para un estudio de los aspectos teórico-metodológicos de las trayectorias laborales. Revista Colombiana de Sociología, 35(1), 127-149.

Rubilar, G. (2013). Repertorios y aproximaciones biográfico-narrativas. Testimonios y análisis de prácticas investigativas en trabajadores sociales. Forum Qualitative Sozialforschung, 14(2).

Serrano, A., Revilla, J., y Arnal, M. (2016). Narrar con imágenes: entrevistas fotográficas en un estudio comparado de "resiliencia" social y resistencia ante la crisis. Empiria, 35, 71-104. https://doi.org/DOI/empiria.34.2016.16526

Sousa, A. A. (2010). El constructivismo estructuralista: La teoría de las clases sociales de Pierre Bourdieu. Reis, 1(75), 145. https://doi.org/10.2307/40184032

Torche, F. (2015). Movilidad intergeneracional y desigualdad: El caso latinoamericano. The Annual Review of Sociology, 40. https://doi.org/10.1146/annurev-soc-06221 5-092006

Torche, F., y Wormald, G. (2004).Estratificacion y Movilidad Social en Chile. Retrieved from https://repositorio.cepal.org/bitstream/handle/11362/6089/S0410803_e s.pdf? sequence $=1 \&$ isAllowed $=y$

Torns, T., Moreno, S., Grau, A., Carrasquer, P., Castelló, L., y Borràs, V. (2011). Trayectorias Laborales y de Vida: Una aproximación al modelo de empleo Español (No. 17). Retrieved from http://quit.uab.es

\section{Notas}

1 Este trabajo es financiado por la Agencia Nacional de Investigación y Desarrollo / Subdirección de Capital Humano / Doctorado Becas Chile/2018 - Folio 72190247. Este trabajo forma parte del proyecto DINAMOS: Dinámicas de movilidad social en España (Referencias PID2019-106548GB-C21 y PID2019-106548GA-C22) financiado por el del Ministerio de Ciencia e Innovación de España, siendo los investigadores principales el Dr. Pedro-López-Roldán y la Dra. Sandra Fachelli (http s://pagines.uab.cat/dinamos/). 
2 Es parte del Proyecto Global trends in social inequalities in Europe and Latin America and exploring innovative ways to reduce them through life, occupational and educational trajectories research to face uncertainty (INCASI: International Network for Comparative Analysis of Social Inequalities), financiado por la European Commission. Horizon 2020 Programme. Marie Skłodowska-Curie Actions (MSCA). Research and Innovation Staff Exchange (RISE).

3 Centre d'estudis sociològics sobre la vida quotidiana i el treball (QUIT), parte del Departamento de Sociología de la Universidad Autónoma de Barcelona. 